\title{
Analysis of vortical structures in a differentially heated lid driven cubical cavity
}

\author{
Hari P. Rani ${ }^{1 *}$, Vekamulla Narayana ${ }^{1}$, Yadagiri Rameshwar ${ }^{2}$ \\ ${ }^{1}$ Department of Mathematics, National Institute of Technology, Warangal 506004, India \\ ${ }^{2}$ Department of Mathematics, College of Engineering, Osmania University, Hyderabad 500007, India
}

Corresponding Author Email: hprani@nitw.ac.in

https://doi.org/10.18280/ijht.360218

Received: 9 January 2018

Accepted: 20 April 2018

Keywords:

mixed convection, Reynolds number,

Richardson number, vortex coreline

\begin{abstract}
Analysis of the vortical structures arising in the system with respect to their control parameters is an important fundamental study. Studies in this regard have mostly been paid attention on a free convective cavity flow. Relatively few studies have been devoted on the characteristics of the vortical structures arising in the mixed convection cavity flows. Thus, it is aimed to analyse the vortical structures arising in a free and forced convective flow of air in a cubical cavity using the direct numerical simulations. Governing equations of this problem, expressed in dimensionless form are solved by using the finite volume method. The simulated results are corroborated with benchmark solutions. Numerical solutions are obtained for wide range of Reynolds number $(R e)$ and Richardson number $(R i)$ (the mixed convection parameter). The flow and thermal characteristics are analysed using isotherms, velocity magnitude, vortex corelines and average Nusselt number. The simulated results show that the large values of $R i$ decrease the total heat transfer rate thus the conductive heat transfer prevails. While when $R i$ takes the small values and for amplified values of $R e$ the complex 3D features are clearly seen and the vigorous forced convection enhances the global heat in the system.
\end{abstract}

\section{INTRODUCTION}

The laminar incompressible mixed convection lid driven cubical cavity flow has wide number of applications in engineering and science such as crystal growth, electronic device cooling, food processing, metal casting and phase change as freezing of water for latent thermal storage systems, solar power collector, glass production etc. Among these numerical experiments some of the benchmark solutions aided to investigate the performance of numerical methodologies and solving the incompressible Navier-Stokes equations for problems with complex geometries. In the literature for the past few decades attention has been focused on mixed convective flow in a cavity in different types of cavity geometries, fluids and imposed temperature gradients.

Moallemi and Jang [1] studied the 2D flow and their thermal features in the laminar flow regime for $100 \leq R e \leq$ 2000 with $0.01 \leq$ Prandtl number $(P r) \leq 50$ and also varying the $R i$ values. The influence of buoyancy on the flow and thermal features is seen to be more aggravated for large values of $\mathrm{Pr}$. It is shown that the free convection assists the forced convection magnitude. A numerical investigation in two dimensional shallow cavities of aspect ratio (AR) 10 was performed by Sharif [2] with heated moving plate. He noticed along the heated moving top wall, the local Nusselt number $(\mathrm{Nu})$ starts with a high value at the left wall and decreases rapidly to a small value towards the right wall. The $\mathrm{Nu}$ in the vicinity of right cold wall shows an oscillatory behaviour because of the existence of a vortex at the cold surface. The average Nusselt number $(\overline{N u})$ augments slowly and quickly with the inclination of the cavity for $R i=0.1$ and
10, respectively. Prasad et al. [3] studied the mixed convective flow inside a $2 \mathrm{D}$ cavity with two vertical side walls kept at a cold temperature and with other vertical walls as adiabatic. It is observed that when the Grashof number $(G r)$ $<0$, a strong convection is manifested for ARs with 0.5 and 1.0. Also it is shown that for $A R=2$, a Hopf bifurcation occurred at $G r=-10^{5}$. In 2D and 3D bottom heating cavities, Mohammad and Viskanta [4] shown that the movement of lid suppresses all forms of convective cells for finite size cavities.

The majority of past literature has been confined to the flow problems in the cavity. While in general, because of the no-slip conditions imposed at the end walls, a closed finite cavity problem possesses the 3D characteristics. Koseff and Street [5-7] observed that the lab experiments on these driven cavity flows have been paid less attention. They conducted experiments to describe the eminent features of the 3D lid driven cavity flows. Using pseudo-spectral method $\mathrm{Ku}$ et al. [8] attempted to compute the flow inside a cubic cavity at $R e$ $=100,400$ and 1000. A systematic computational exercise was performed by Iwatsu et al. [9-11] for a cubical cavity in $100 \leq R \mathrm{e} \leq 4000$. One among the main result of these 3D numerical simulations indicates that the steady solutions are attained at lower values of $R e$, but the flow becomes unsteady when $R e$ exceeds approximately 2000. Aydin et al. [12] numerically analysed the transport mechanism of free and forced convection in a shear cavity with bottom heated wall and other walls as sliding.

From the previous studies, it can be observed that for the flow in the 2D cavity in the absence of top lid motion the vertical heat transfer is shown to be entirely conductive and the externally applied temperature difference is 
gravitationally stable. But for the flow in the 3D cavity, it is analysed that due to the movement of the lid, the mechanically driven convection is induced along with the enhancement of associated 3D heat movements. Thus along with imposed conditions on the cavity in realistic applications, the 3D constraints have to be taken into consideration. Thus, in the present paper the $3 \mathrm{D}$ features in the cubical cavity are aimed to analyse. The structure of 3D flows using the comprehensively and systematically organized numerical simulations by varying the control parameters is presented.

The heat transfer mechanism in a lid driven cubical cavity by augmenting the convective activities is analysed in the steady state region of $100 \leq R e<2000,0 \leq R i \leq 10$ with $P r=$ 0.71 . These considered parameter values are inline with Iwatsu et al. [11] and Ouertatani et al. [14]. The previous works of [1-12] is extended to analyse the vortical structures arising in this system. Using the open source software, namely, OpenFOAM the simulations are carried out. The finite volume method is used to discretize the governing equations. The enormous output data is analysed with help of velocity magnitude, isotherms, vortical corelines etc.

The paper is organised as follows: In section 2, the configuration of the considered geometry along with the mathematical model is given. The adopted methodology to solve the model is explained. The simulated results are validated extensively and shown in section 3 . The simulated results are analysed with respect to streamlines, isotherms and vortex corelines and given in section 4 . The last section summarises the work done in this paper.

\section{PHYSICAL SYSTEM AND GOVERNING EQUATIONS}

A cubical lid driven cavity of length $L$ is considered in the Cartesian coordinate $(X, Y, Z)$ system which is filled with air. The schematic of the considered geometry along with the boundary conditions are shown in Fig. 1.

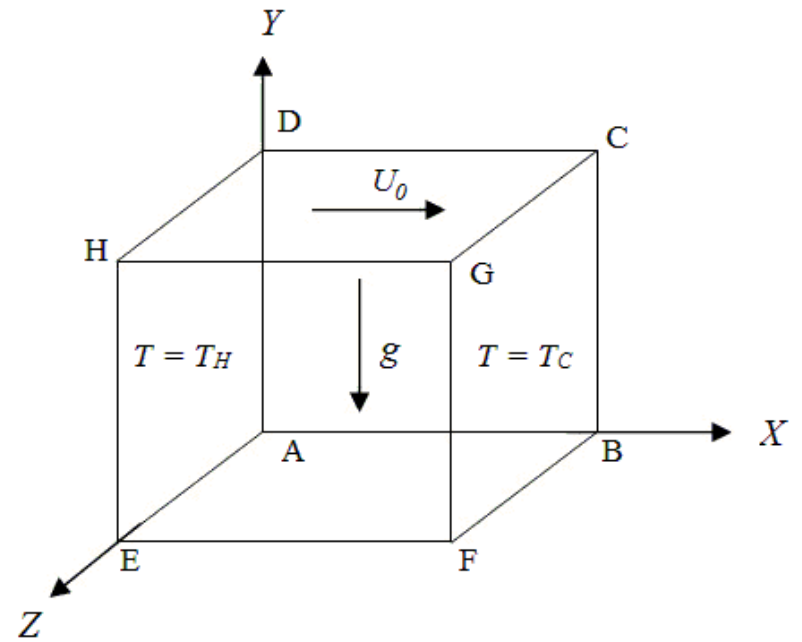

Figure 1. Schematic of lid driven 3D cavity of length $L$

The top lid situated at $Y=L$ moves steadily in its own parallel plane with a constant velocity $\left(U_{0}\right)$, while the other boundary walls are kept at rest. The left side wall is assumed to be kept at a higher temperature $\left(T_{H}\right)$ than that of the right side wall $\left(T_{C}\right)$ with difference in temperature $\left(\Delta T=T_{H}-T_{C}\right)>$
0 . In addition to these assumptions, the other four remaining boundary walls are assumed to be adiabatic.

The considered 3D physical system is converted to a mathematical model by the steady laminar dimensionless form for the mass conservation, momentum and energy equations with the Boussinesq approximations as

$\operatorname{div} \boldsymbol{V}=0$

$(\boldsymbol{V} \cdot \operatorname{grad}) \boldsymbol{V}=-\operatorname{grad} p+\frac{1}{R e} \cdot \nabla^{2} \boldsymbol{V}+R i . T^{*} \boldsymbol{e}$

$($ V. $\operatorname{grad}) T^{*}=\frac{1}{R e \cdot \operatorname{Pr}} \cdot \nabla^{2} T^{*}$

where notations are explained in the Nomenclature. The above equations (1)-(3) are closed with the following boundary conditions:

$\boldsymbol{V}=(1,0,0)$ at $Y=1 ; \boldsymbol{V}=0$ at $Y=0, X=0,1 ; Z=0,1$

$T^{*}=1$ at $X=0 ; T^{*}=0$ at $X=1$.

$\frac{\partial T^{*}}{\partial Y}=0$, at $X=0,1$ and $\frac{\partial T^{*}}{\partial Z}=0, Z=0,1$

The non-dimensional heat transfer rate $(N u)$ at the hot wall is computed is by $N u=\left(\frac{\partial T^{*}}{\partial X}\right)_{X=0}$. The average heat transfer rate $(\overline{N u})$ is obtained by integrating $N u$ along the hot wall.

With the aid of above numerical model given in Eqs. (1)(3) the open source software namely, the OpenFOAM is used to simulate the fluid flow inside the cubical cavity. In the buoyantBoussinesqSimpleFoam of the CFD solver the geometry, flow volume and boundary conditions are set. It is a steady state solver for buoyant flow of incompressible fluid that includes Boussinesq approximation. The second order upwind linearization technique is employed for the spatial derivatives. The CGS method was used as an effective acceleration means and divergent and Laplacian terms are discretized by the QUICK and Gauss linear schemes, respectively.

\section{VALIDATION}

Table 1. Validation of simulated results with respect to $\overline{N u}$

\begin{tabular}{|c|c|c|c|c|}
\hline \multirow{3}{*}{$R e$} & & 0.001 & 1 & 10 \\
\hline \multirow{4}{*}{100} & & & & \\
\hline \multirow{3}{*}{400} & Present work & 1.8134 & 1.305 & 1.075 \\
\cline { 2 - 5 } & Iwatsu et al.[11] & 1.820 & 1.330 & 1.080 \\
\cline { 2 - 5 } & Ouertatani et al. [14] & 1.836 & 1.348 & 1.092 \\
\cline { 2 - 5 } & Present work & 3.880 & 1.540 & 1.169 \\
\cline { 2 - 5 } 1000 & Iwatsu et al. [11] & 3.990 & 1.500 & 1.170 \\
\cline { 2 - 5 } & Ouertatani et al. [14] & 3.964 & 1.528 & 1.130 \\
\cline { 2 - 5 } & Present work & 7.150 & 1.790 & 1.340 \\
\cline { 2 - 5 } & Iwatsu et al. [11] & 7.030 & 1.800 & 1.370 \\
\cline { 2 - 5 } & Ouertatani et al. [14] & 7.284 & 1.856 & 1.143 \\
\hline
\end{tabular}

The present simulated results are corroborated with the similar works done by Iwatsu et al. [9] and Nasreddine Ouertatani et al. [11] in terms of $\overline{N u}$. The validation is performed for $R i=0.001,1$ and 10 and $R e=100,400$ and 1000 and the values are tabulated in Table 1. The deviation between the present simulated results and the previous results is very less. Thus, the data obtained from the present study is 
used to predict the characteristics of mixed convective heat transfer in the cubical cavity.

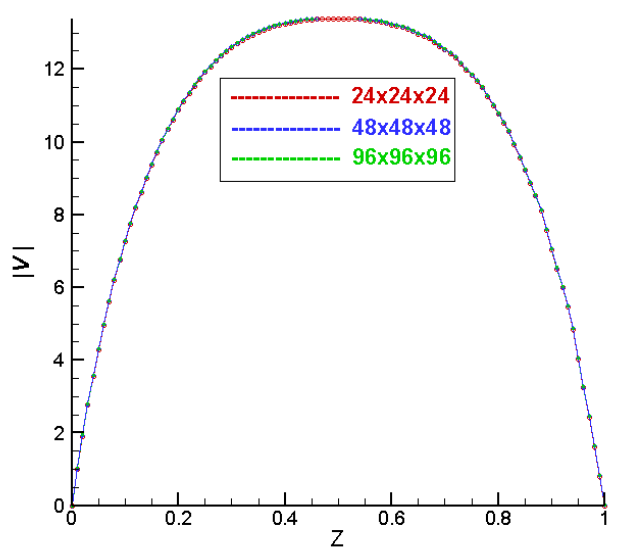

(a) Velocity magnitude $|\boldsymbol{V}|$ at $X Y$ mid plane

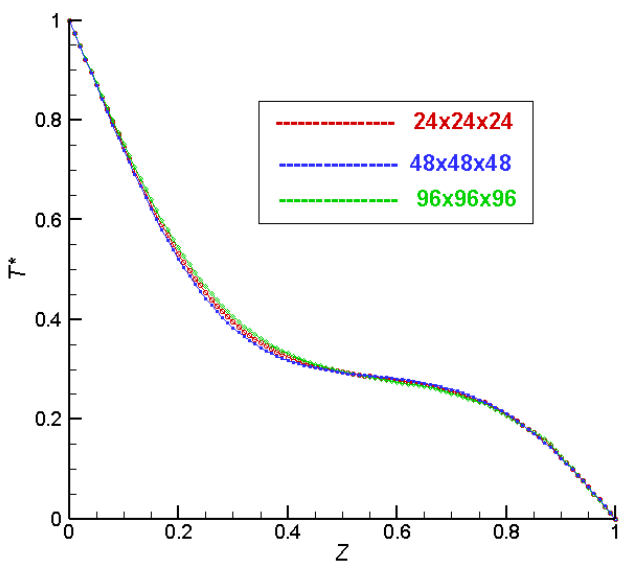

(b) Temperature $T^{*}$ at $X Y$ mid plane

Figure 2. Grid independent test for $R e=100, R i=0.001$.

Figure 2 shows the $|\boldsymbol{V}|$ and $T^{*}$ solutions calculated using three different size meshes, namely $24^{3}, 48^{3}$ and $96^{3}$ with $R i$ $=0.001$ and $R e=100$. Figures 2 (a) and (b) show $|\boldsymbol{V}|$ and $T^{*}$ in the $X Y$ mid plane, respectively, that are calculated using the above different mesh sizes. From Fig. 2 it is observed that currently employed $48^{3}$ uniformly stretched mesh results did not show much difference from those when the mesh resolution was increased and decreased by $50 \%$. Hence the mesh with $48^{3}$ resolutions was used for the simulations which are conducted in the investigated domain.

\section{RESULTS AND DISCUSSION}

The simulated results are plotted as streamlines and isotherms for different control parameter values, such as $R e$ and $R i$ arising in the system. The important aspects of combined effect of $R e$ and $G r$ on the vortical structures is analyzed. The physical parameters $R e$ and $G r$ control the flow characteristics and hence they are varied proportionally so that the mixed convection parameter $R i$ is kept constant as $R i<1,=1$ and $>1$. For these $R i$ values, from the Table 1 it can be observed that the $\overline{N u}$ gets augmented with $R e$ while it gets diminished for increasing values of $R i$ and the variation between each value is more that $50 \%$.
For the past few decades extensive experimental and numerical investigations have paid attention on the recirculation phenomena arising inside the cavity along with the developing and deforming 3D vortical structures and coherent structures. Three major regions in the cavity classify the flow field are: (i) the flow near the hot wall (ii) centre of the cavity where the energy exchange takes place through the interaction of the vortices and (iii) the vicinity of the lid, where there is a large velocity gradient exists due to the movement of lid. Thus, the analysis of vortex dynamics of this flow is a demanding task.

\subsection{Isotherms}

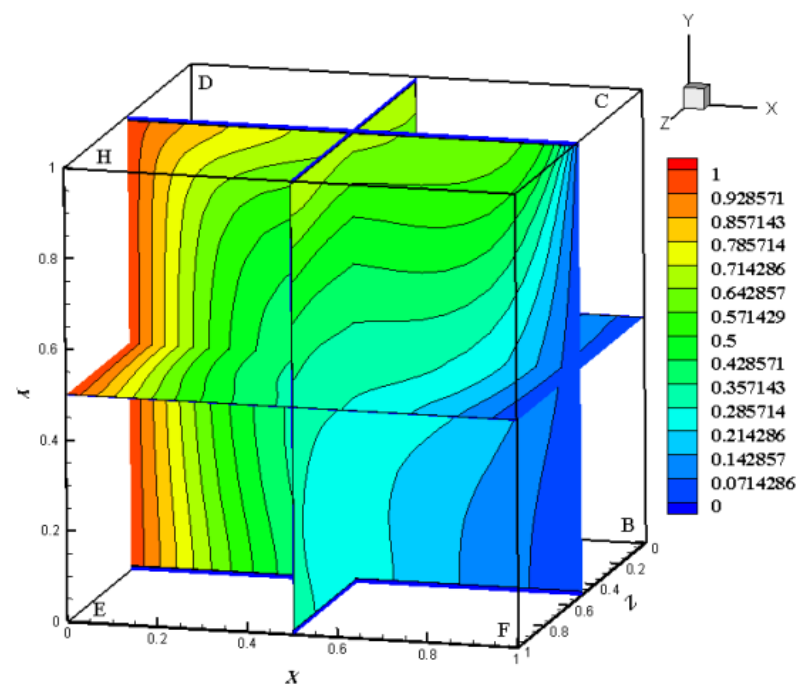

(a) $R e=100, R i=0.001$

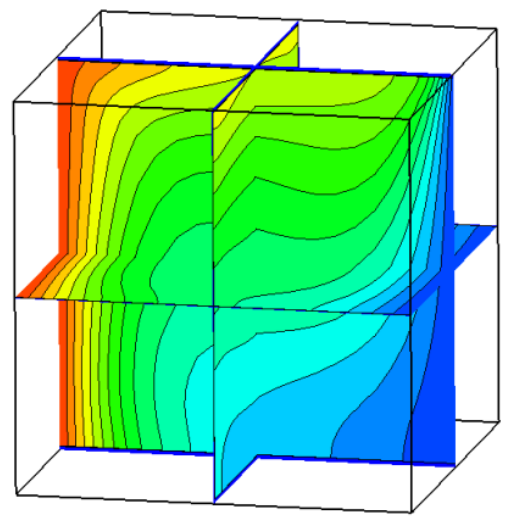

(b) $R e=100, R i=1$

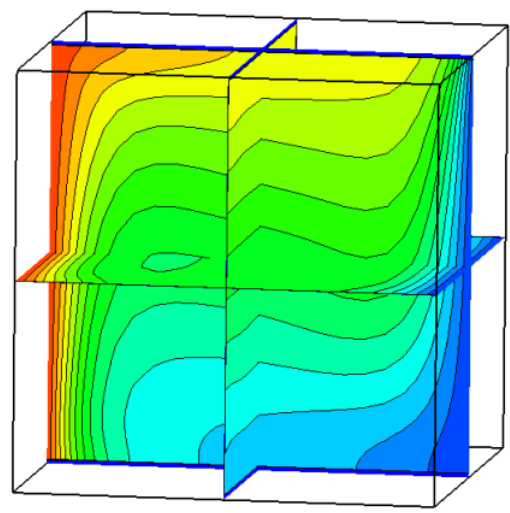

(c) $R e=100, R i=10$ 


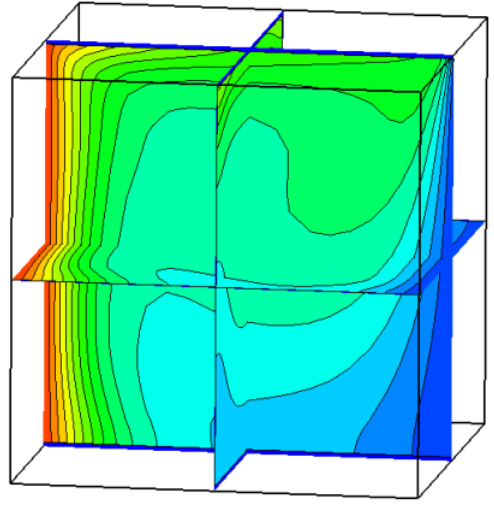

(d) $R e=400, R i=0.001$

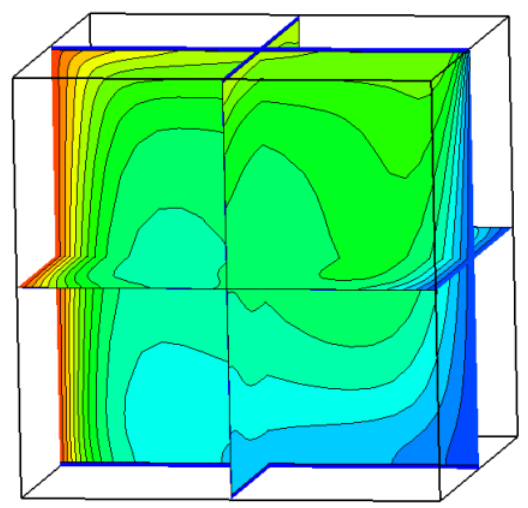

(e) $R e=400, R i=1$

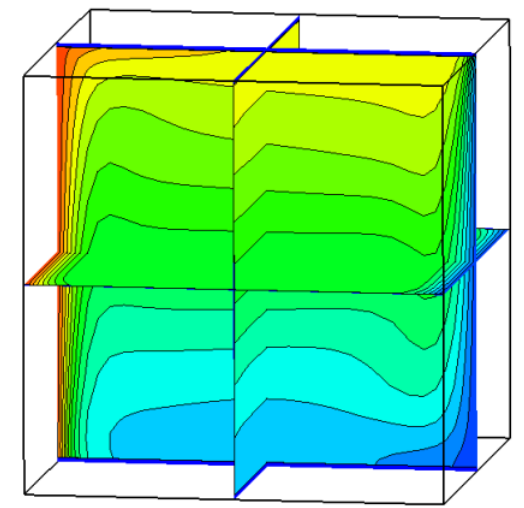

(f) $R e=400, R i=10$

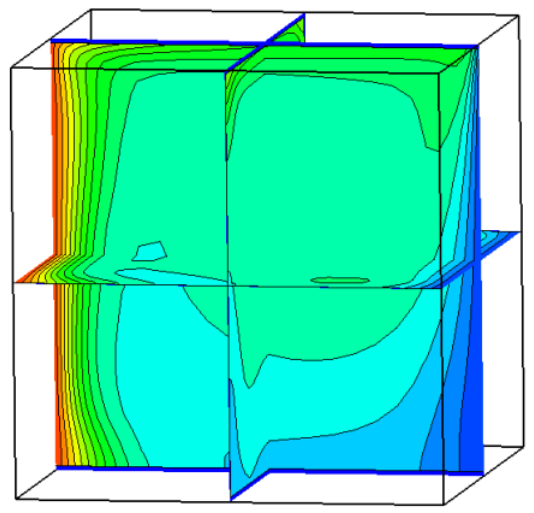

(g) $R e=1000, R i=0.001$

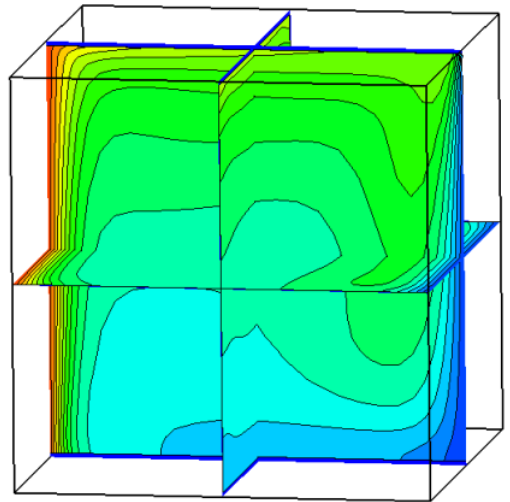

(h) $R e=1000, R i=1$

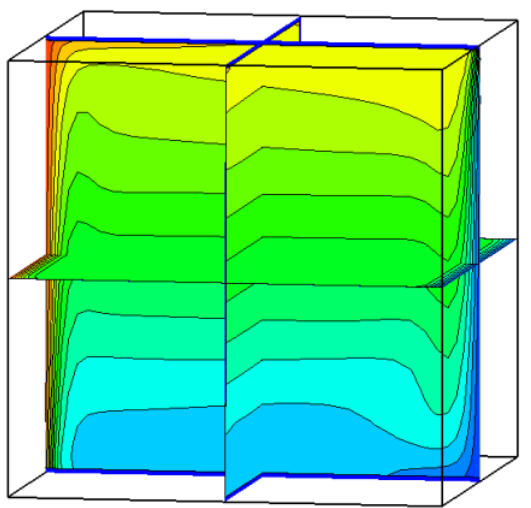

(i) $\operatorname{Re}=1000, R i=10$

Figure 3. Isotherms for different $R i$ and $R e$. The contour legend used in Figs. (a)-(i) is shown in Fig (a)

The influence of $R e$ and $R i$ on the temperature contours is illustrated in Fig. 3. When $R i$ is fixed at 0.001 and $R e$ increases from 100 to 1000 , i.e., as velocity of the upper lid increases, it is observed that vertical isotherms start to appear. The buoyancy driven convection is dominated by the mechanically driven forced convection as observed from Figs. 3(a, d, g). This result implies the lid movement is due to the forced convection only. As the isotherms depart from the vertical position, i.e. $R i=1$, there is a change in the heat transfer mechanism between the conduction and convection states. Initially the isotherms at the centre of the cavity are horizontal and become vertical especially inside the very thin boundary layers as shown in Figs. 3(b, e, h). While on the other hand, when $R i$ augments from 1 to 10, isotherm fields get distorted by the buoyant convection and $3 \mathrm{D}$ patterns become more pronounced as shown in Figs. 3(c, f, i). The isotherm field gets more distorted with increasing $R i$ values. This is due to the fact that if the forced convection due to the lid movement is almost nil, buoyancy force dominates the flow and natural convection controls the heat transfer.

\subsection{Velocity magnitude}

Figure 4 shows the velocity magnitude distributions on the symmetry planes for different $R e$ and $R i$. We observe that the flow motion in $3 \mathrm{D}$ cavity is elaborated due to the effect of lateral walls. For the cases of low $R e$ flow tunnels are developed near the center of walls, and as $R i$ increases, they 
move to the corners of the cavity. As shown in Fig. 4(c), the maximum velocity is located adjacent to two corners of the isothermal walls, which means the two tunnels have been developed there. In addition, we also find that similar to the temperature field, velocity boundary layers are generated close to the isothermal walls, which become thinner with increasing $R e$, but no apparent velocity boundary layers are developed adjacent to the adiabatic walls. This result indicates that contrary to those results obtained near the adiabatic wall i.e., a boundary layer is formed adjacent to the isothermal wall. This outcome is consistent with the phenomenon observed in Fig. 4.

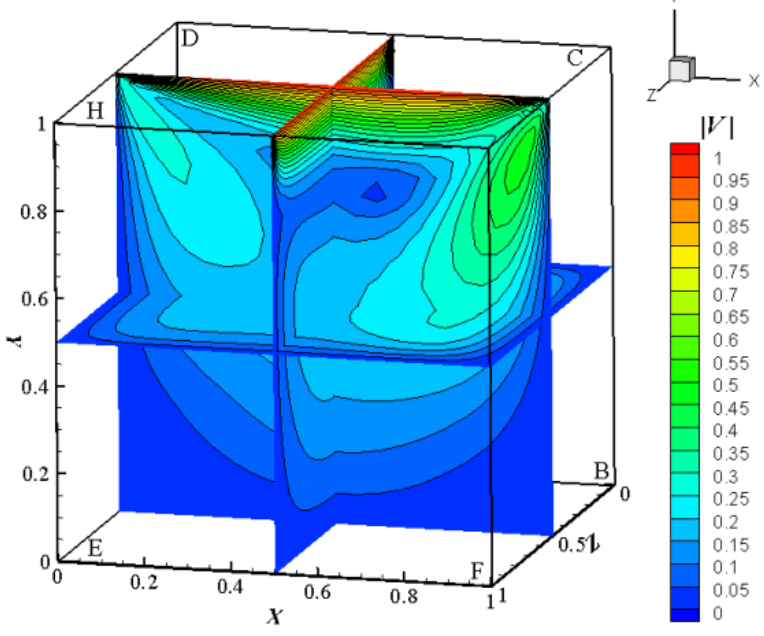

(a) $R e=100, R i=0.001$

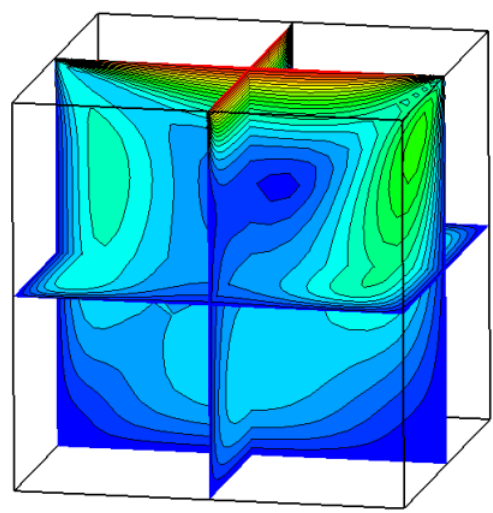

(b) $R e=100, R i=1$

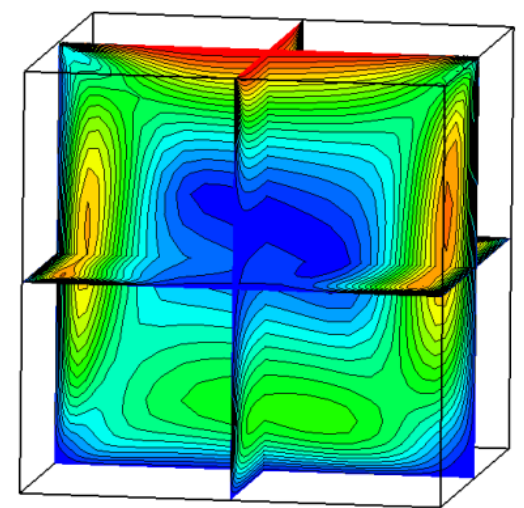

(c) $R e=100, R i=10$

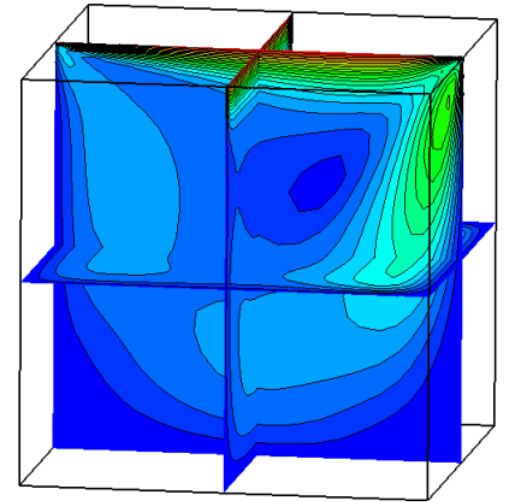

(d) $R e=400, R i=0.001$

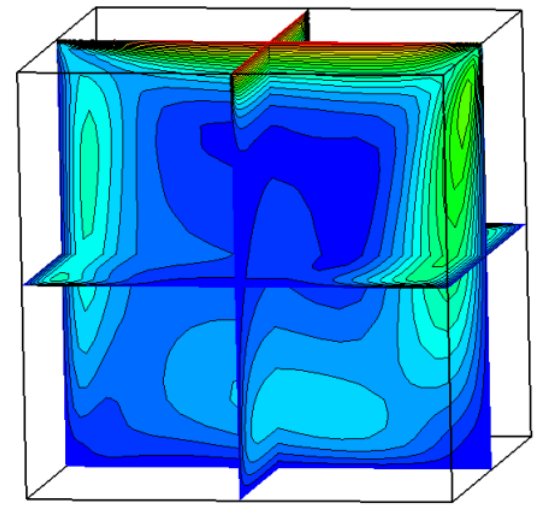

(e) $R e=400, R i=1$

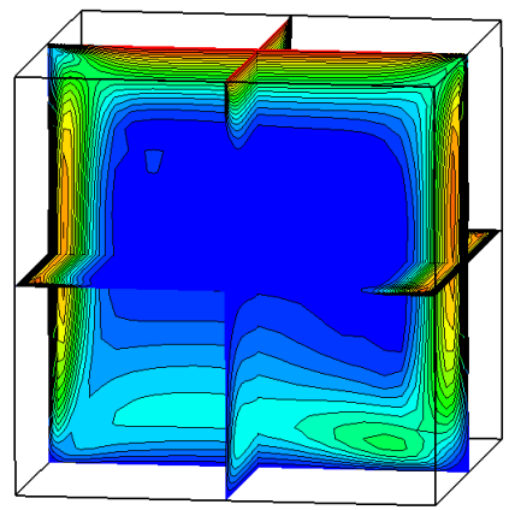

(f) $R e=400, R i=10$

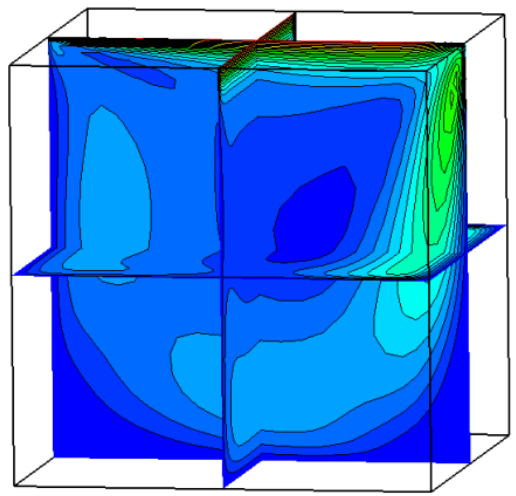

(g) $R e=1000, R i=0.001$ 


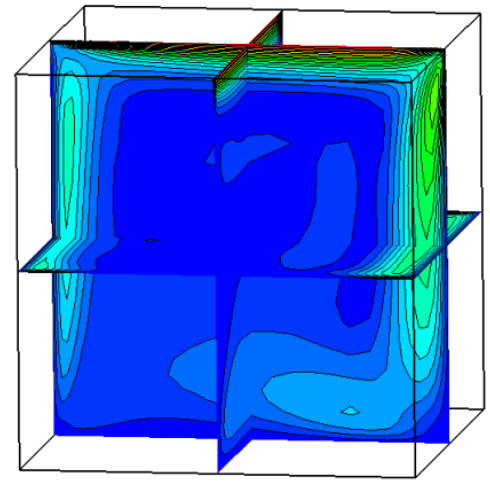

(h) $R e=1000, R i=1$

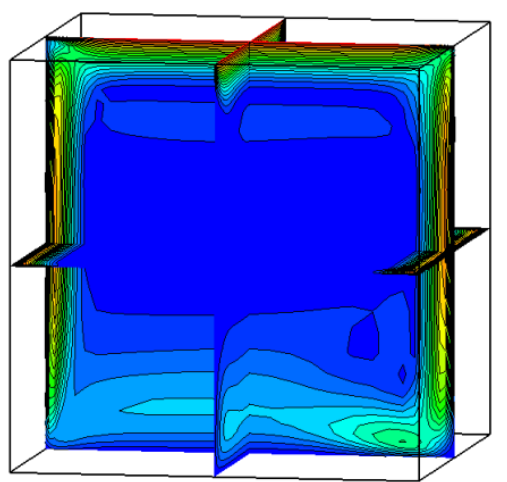

(i) $R e=1000, R i=10$

Figure 4. Velocity magnitude for different $R i$ and $R e$ The contour legend for Figs. (a)-(i) is shown in Fig.(a)

\subsection{Vortex corelines and streamlines}

The swirling motion in the fluid is regarded as the vortex and the main signature of the vortical flow is given by the vortex corelines. According to Robinson [15] the vortex is defined as "A vortex exists when instantaneous streamlines mapped onto a plane normal to the vortex core exhibit a roughly circular or spiral pattern, when viewed from a reference frame moving with the centre of the vortex core". Thus, an analysis is made from the enormous simulated data to understand the signature of vortex flow with respect to the different control parameters. The vortices that generated from the end walls are detected and visualized. In general these vortices consist of critical points in the wall shear stress vector field [16].

Figure 5 depicts the vortex corelines and swirling streamlines around these corelines for $R e=100$ and $R i=$ 0.001 . The velocity gradient eigen-mode method was used to extract these vortex corelines. The front and right side views of the cavity are shown to clearly visualize the vortical structures arising in the system. Also Fig. 5 shows the pattern of vortical coreline that occur in the recirculation region. The coreline depicts the complex surface streaking structure. Since the fluid particles near the endwall lead the vortical motion to proceed along the third dimension, the vortex motion shows its start/end in the bounding endwalls [13]. Also the fluid particles about this line have an inclination to move spirally towards the symmetry plane. At the centre of the cavity there are two vortices that are similar and interact with each other. The exchange of energy among these two closed vortical structures happens at the centre of the cavity. Hence the 3D flow structure is necessary to understand the realistic nature of flow.

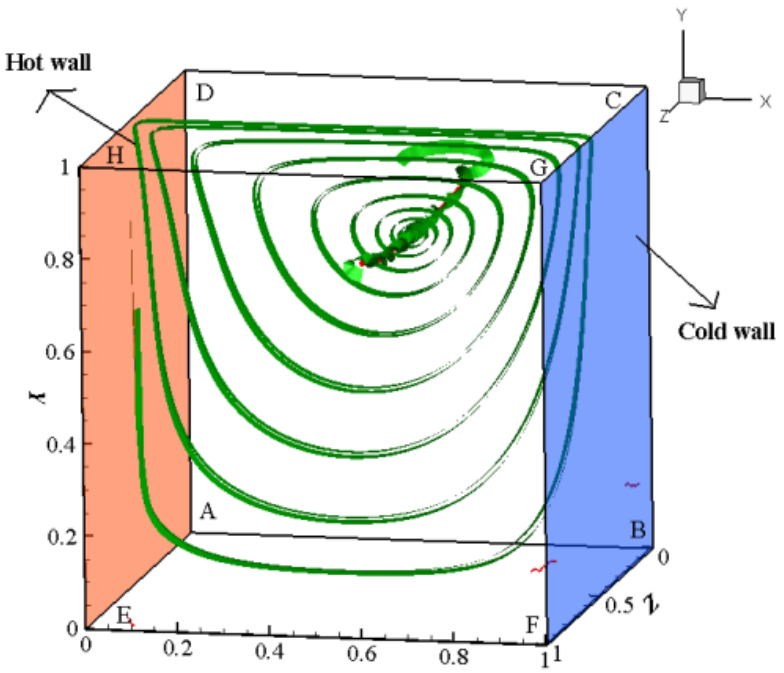

(a) Front view

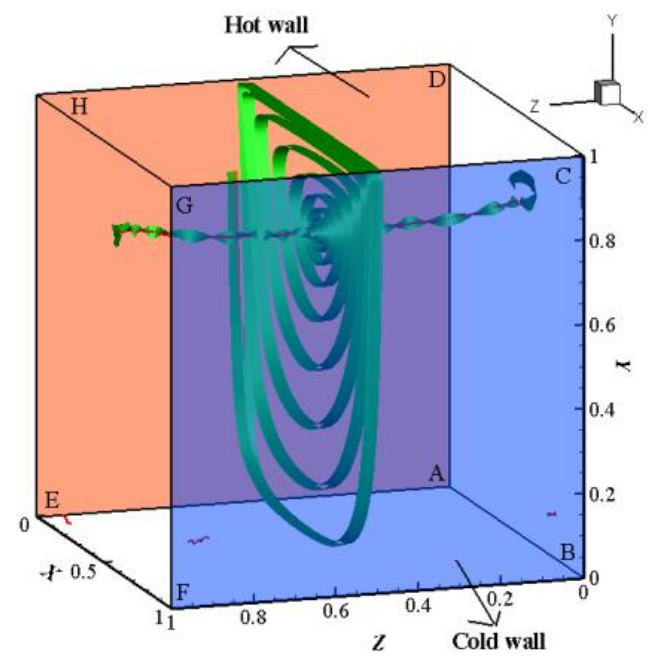

(b) Viewing from right side

Figure 5. Vortex corelines and Streamlines for $R e=100$ and $R i=0.00$. The red and blue denote the hot and cold walls respectively. The streamtraces are shown in green color

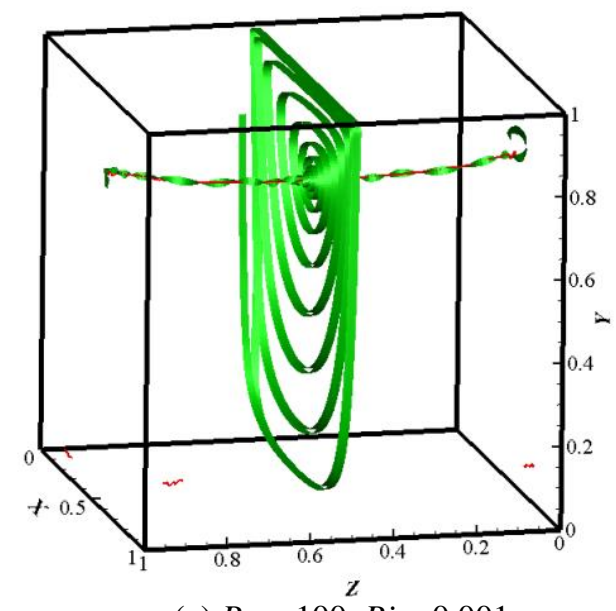

(a) $R e=100, R i=0.001$ 


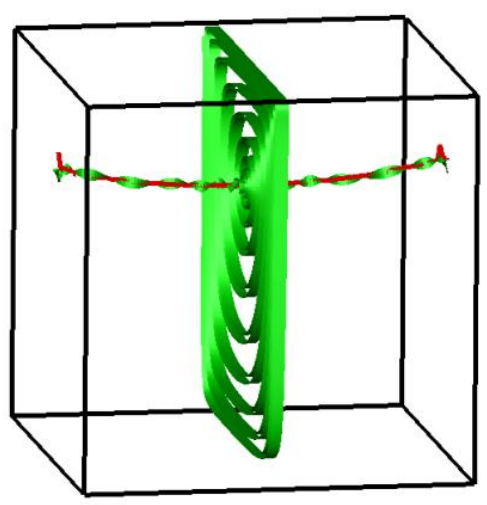

(b) $R e=100, R i=1$

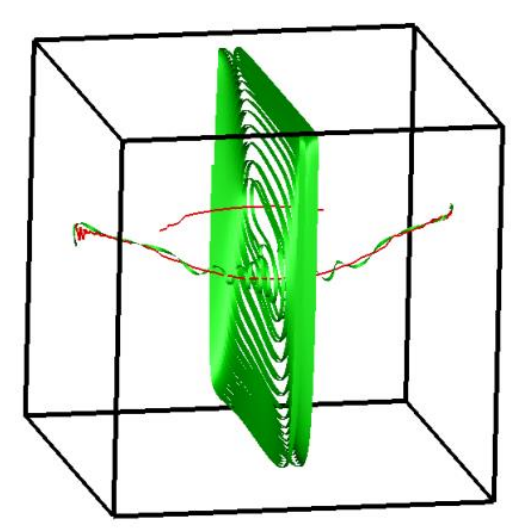

(c) $R e=100, R i=10$

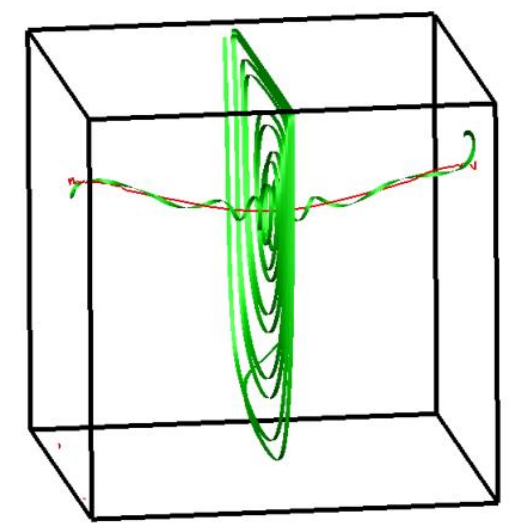

(d) $R e=400, R i=0.001$

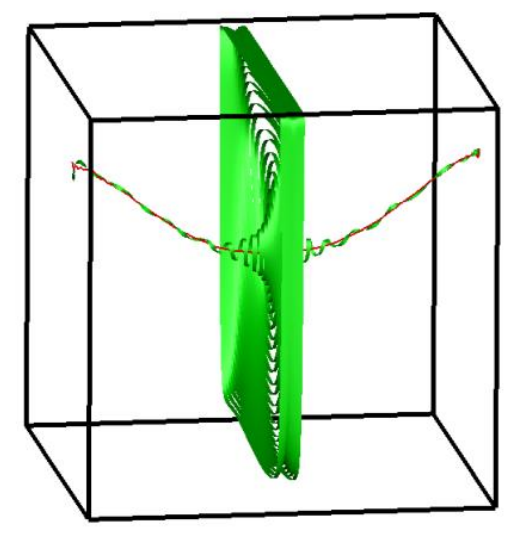

(e) $R e=400, R i=1$

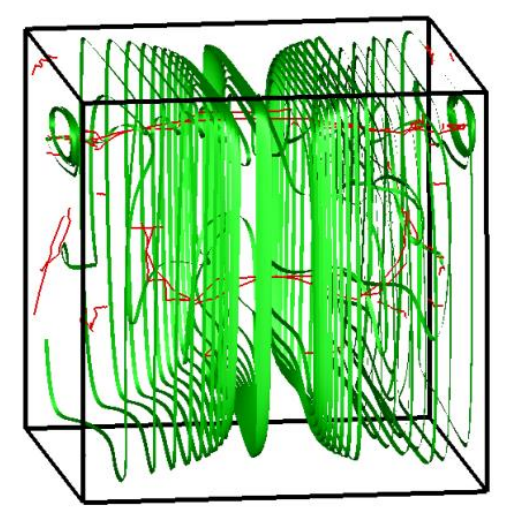

(f) $R e=400, R i=10$

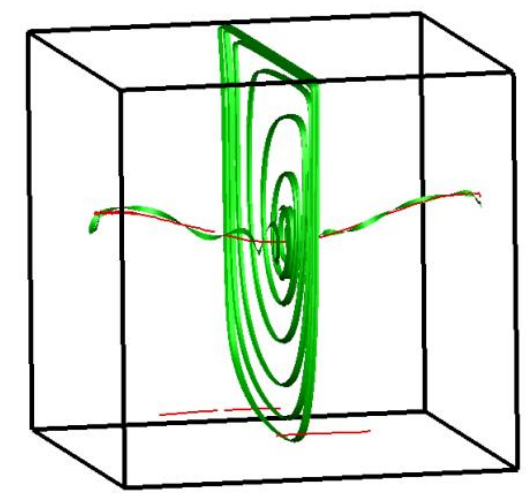

(g) $R e=1000, R i=0.001$

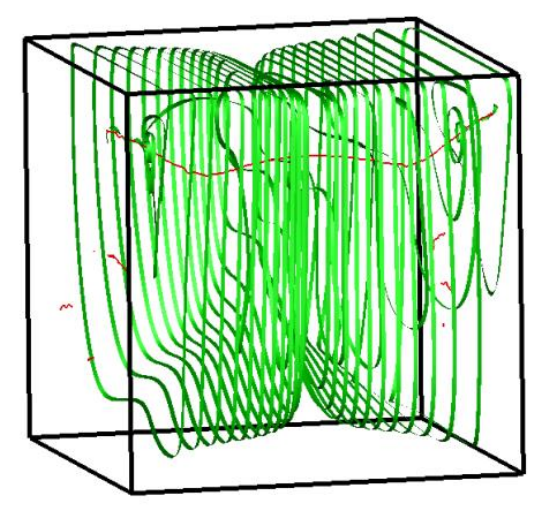

(g) $R e=1000, R i=1$

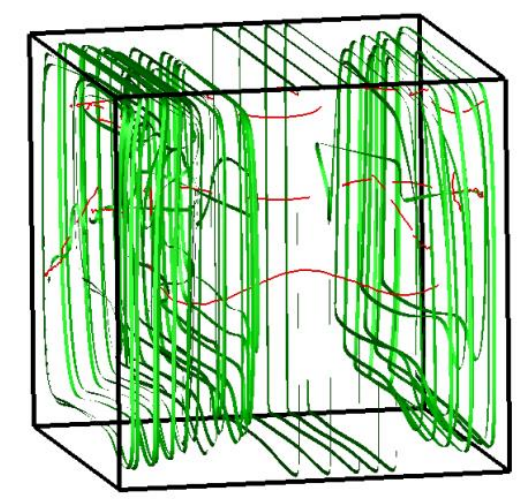

(i) $R e=1000, R i=10$

Figure 6. Visualizing vortex corelines (red color) and Streamtraces (green color) for different $R i$ and $R e$ 
Figure 6 illustrates the streamlines and the vorticity in terms of vortical corelines in the cubical cavity for different combinations of $R e$ and $R i$ values. The swirling nature is closer to the vortex lines as $R i$ increases. The strength of the vorticity increases as $R e$ or $R i$ increases. When $R i<0.1$ the natural convection is insignificant, $R i>10$ forced convection is insignificant, and for $0.1<R i<10$ neither free nor forced convection are significant. The forced convection is large relative to natural convection, except in the case of extremely low forced flow velocities.

The following three different cases of $\mathrm{Ri}$ are investigated in the present study: (i) $R i(=0.001)$ is much less than unity (free convection); (ii) $R i$ is of order unity $(=1)$, where the flow is buoyancy-driven or the energy of the flow derived from the potential energy in the system (mixed convection); (iii) $R i \gg 1(=10)$, i.e., the buoyancy is dominant or to homogenize the fluids, there is insufficient kinetic energy (forced convection). The above three cases are depicted in Figs. 6(a, d, g), Figs. 6(b, e, h) and Figs. 6(c, f, i), respectively. It can be observed that for all values of $R i$ the increasing values of $R e$ from 100 to 1000 shows the chaotic nature of the flow from laminar to transitional. As $R e$ increases the streamlines are much distorted. The flow behaviour observed to be different in different types of convection dominated regimes.

\section{CONCLUSION}

In the present paper an attempt is made to address the $3 \mathrm{D}$ laminar free and forced convective flow in a cubical cavity filled with air for the non-dimensional numbers $R e$ and $R i$ arising in the system. The effects of these parameters and their resulting convection disturbances are investigated. When $R i<<1$, the isotherm surfaces maintain a two dimensional structures for $R e=100$ and 3D structures are visualised when $R e$ takes the large values. But for the large $R i$ values there is a stabilizing buoyancy effect, heat transfer is largely by convection and three dimensionality in the thermal field is weak. When $R i>>1$, the heat transfer rate is suppressed along with the conductive heat transfer. It is observed that vigorous forced convection enhances the global heat.

\section{REFERENCES}

[1] Moallemi MK, Jang KS. (1992). Prandtl number effects on laminar mixed convection heat transfer in a liddriven cavity. Int. J. Heat Mass Transfer 35: 1881-1892. http://doi.org/10.1016/0017-9310(92)90191-t

[2] Sharif MAR. (2007). Laminar mixed convection in shallow inclined driven cavities with hot moving lid on top and cooled from bottom. Appl. Ther. Eng. 27: 10361042. 10.1016/j.applthermaleng.2006.07.035

[3] Prasad YS, Das MK. (2007). Hopf bifurcation in mixed flow inside a rectangular cavity. Int. J. Heat Mass Transfer 50: 3583-3598. http://doi.org/ 10.1016/j.ijheatmasstransfer.2006.11.048

[4] Mohammad AA, Viskanta R. (1992). Laminar flow and heat transfer in Rayleigh-Benard convection with shear. Phys. Fluids 4: 2131-2140. http://doi.org/ 10.1063/1.858509

[5] Koseff JR, Street RL. (1984). Visualization studies of a shear driven three dimensional recirculating flow. J. Fluids Eng. 106: 21-29. http://doi.org/ $10.1115 / 1.3242393$

[6] Koseff JR, Street RL. (1984). On end wall effects in a lid driven cavity flow. J. Fluids Eng. 106: 385-389. http://doi.org/10.1115/1.3243135

[7] Koseff JR, Street RL. (1984). The lid-driven cavity Flow: A synthesis of qualitative and quantitative observations. J. Fluids Eng. 106: 385-389. http://doi.org/10.1115/1.3243136

[8] Ku HC, Hirish RS, Taylors TD. (1987). A Pseudospectral Method for solution of the ThreeDimensional Incompressible Navier-Stokes equations. J. Comput. Phys. 70: 439-462. http://doi.org/10.1016/0021-9991(87)90190-2

[9] Iwatsu R., Ishii K, Kawamura T, Kuwahara K, Hyun JM. (1989). Numerical simulation of three dimensional flow structure in a driven cavity. Flu. dynamic. Research 5:173-189. http://doi.org/10.1016/01695983(89)90020-8

[10] Iwatsu R, Hyun JM, Kuwahara K (1989). Analyses of three dimensional flow calculations in a driven cavity. Flu. dynamic. Research 6: 91-102. http://doi.org/10.1016/0169-5983(90)90030-3

[11] Iwatsu R, Hyun JM. (1995). Three dimensional drivencavity flows with a vertical temperature gradient. Int. J. Heat Mass Transfer 38: 3319-3328. http://doi.org/10.1016/0017-9310(95)00080-s

[12] Aydin O, Yang WJ. (2000). Mixed convection in cavities with a locally heated lower wall and moving sidewalls. Numer. Heat Trans, Part A: Applications 37: 695-710. http://doi.org/10.1080/104077800274037

[13] Benkacem N, Cheikh NB, Beya BB. (2015). Threedimensional analysis of mixed convection in a differentially heated lid-driven cubic enclosure. J Appl. Mech. Eng. 4: 159-162. http://doi.org/10.4172/21689873.1000159

[14] Ouertatani N, Cheikh NB, Beya BB, Taieb L, Antonio C. (2009). Mixed convection in a double lid-driven cubic cavity. Int. J. Thermal Sciences 48: 1265-1272. http://doi.org/10.1016/j.ijthermalsci.2008.11.020

[15] Robinson SK. (1991). Coherent motions in the turbulent boundary layer. Annual Review of Fluid Mechanics 23: 601-639. http://doi.org/10.1146/annurev.fl.23.010191.003125

[16] Tony Sheu WH, Rani HP, Tan TC, Tsai SF. (2008). Multiple states, topology and bifurcations of natural convection in a cubical cavity. Comput. and Fluids 37: 1011-1028. http://doi.org/10.1016/j.compfluid.2007.11.003

\section{NOMENCLATURE}

$g$

Gr

$L$

$\frac{N u}{N u}$

$\overline{N u}$

$p$

$\operatorname{Pr}$ gravitational acceleration $\left(\mathrm{ms}^{-2}\right)$ Grashof number $\left(=\frac{g \beta(\Delta T) L^{3}}{v^{3}}\right)$

Length of the cavity (m)

Local Nusselt number

Average Nusselt number

non-dimensional pressure

Prandtl number $\left(=\frac{v}{\alpha}\right)$ 
$R a$

Re

$R i$

$T$

$T^{*}$

$U_{0}$

$V$

$X, Y, Z$
Rayleigh number $\left(=\frac{g \beta(\Delta T) L^{3} P r}{v^{3}}\right)$

Reynolds number $\left(=U_{0} h / v\right)$

Richardson number ( $=G r \cdot R e^{-2}$

Dimensional temperature $(\mathrm{K})$

Dimensionless temperature $\left(=\frac{T-T_{C}}{\Delta T}\right)$

Top wall velocity $\left(\mathrm{m} \mathrm{s}^{-1}\right)$

Dimensionless velocity vector

Cartesian coordinates (m)

\section{Greek symbols}

$\alpha$

$\beta$

$v$

$\rho$

$\Delta T$

Subscripts

C

H coefficient of thermal diffusion $\left(\mathrm{m}^{2} \mathrm{~s}^{-1}\right)$ coefficient of thermal expansion $\left(\mathrm{K}^{-1}\right)$ molecular kinematic viscosity $\left(\mathrm{m}^{2} \mathrm{~s}^{-1}\right)$ density

temperature difference, $\left(=T_{H}-T_{C}>0\right)$
Cold wall

Hot wall

mean 